\title{
Clinical implications of Mycobacterium chimaera detection in thermoregulatory devices used for extracorporeal membrane oxygenation (ECMO), Germany, 2015 to 2016
}

FC Trudzinski ${ }^{1}$, U Schlotthauer ${ }^{2}$, A Kamp ${ }^{1}$, K Hennemann ${ }^{3}$, RM Muellenbach ${ }^{4}$, U Reischl 5 , B Gärtner ${ }^{2}$, H Wilkens ${ }^{1}$, R Bals ${ }^{1}$,

M Herrmann ${ }^{26}$, PM Lepper ${ }^{1}$, SL Becker ${ }^{278}$

1. Department of Medicine V - Pneumology, Allergology and Critical Care Medicine, ECLS Center Saar, Saarland University, Homburg/Saar, Germany

2. Institute of Medical Microbiology and Hygiene, Saarland University, Homburg/Saar, Germany

3. Department of Thoracic and Cardiovascular Surgery, Saarland University, Homburg/Saar, Germany

4. Department of Anaesthesiology and Critical Care, Campus Kassel of the University Hospital of Southampton, Kassel, Germany

5. Institute of Clinical Microbiology and Hygiene, University Hospital Regensburg, University of Regensburg, Regensburg, Germany

6. Faculty of Medicine, University of Münster, Münster, Germany

7. Swiss Tropical and Public Health Institute, Basel, Switzerland

8. University of Basel, Basel, Switzerland

Correspondence: Sören L. Becker (soeren.becker@uks.eu) and Philipp M. Lepper (philipp.lepper@uks.eu)

Citation style for this article:

Trudzinski FC, Schlotthauer U, Kamp A, Hennemann K, Muellenbach RM, Reischl U, Gärtner B, Wilkens H, Bals R, Herrmann M, Lepper PM, Becker SL. Clinical implications of Mycobacterium chimaera detection in thermoregulatory devices used for extracorporeal membrane oxygenation (ECM0), Germany, 2015 to 2016. Euro Surveill. 2016;21(46):pii=30398. DOI: http://dx.doi.org/10.2807/1560-7917.ES.2016.21.46.30398

Article submitted on 07 September 2016 / accepted on 19 October 2016 / published on 17 November 2016

Mycobacterium chimaera, a non-tuberculous mycobacterium, was recently identified as causative agent of deep-seated infections in patients who had previously undergone open-chest cardiac surgery. Outbreak investigations suggested an aerosol-borne pathogen transmission originating from water contained in heater-cooler units (HCUs) used during cardiac surgery. Similar thermoregulatory devices are used for extracorporeal membrane oxygenation (ECMO) and $M$. chimaera might also be detectable in ECMO treatment settings. We performed a prospective microbiological study investigating the occurrence of $M$. chimaera in water from ECMO systems and in environmental samples, and a retrospective clinical review of possible ECMO-related mycobacterial infections among patients in a pneumological intensive care unit. We detected $M$. chimaera in 9 of 18 water samples from 10 different thermoregulatory ECMO devices; no mycobacteria were found in the nine room air samples and other environmental samples. Among 118 ECMO patients, 76 had bronchial specimens analysed for mycobacteria and $M$. chimaera was found in three individuals without signs of mycobacterial infection at the time of sampling. We conclude that $M$. chimaera can be detected in water samples from ECMO-associated thermoregulatory devices and might potentially pose patients at risk of infection. Further research is warranted to elucidate the clinical significance of M. chimaera in ECMO treatment settings.

\section{Introduction}

Mycobacterium chimaera is a slowly growing atypical mycobacterium that is closely related to the more commonly encountered species $M$. avium and $M$. intracellulare [1]. The potential of $M$. chimaera to cause clinical disease was previously considered to be low $[2,3]$, however, a multi-country outbreak of severe infections due to $M$. chimaera was recently described in patients who had undergone open-chest cardiac surgery [4]. Indeed, $M$. chimaera was identified as the causative agent of deep-seated infections such as endocarditis and vertebral osteomyelitis in patients from different European countries (e.g. Germany, the Netherlands, Switzerland) $[5,6]$ and from North America [7]. Interestingly, these infections occurred up to 5 years after the patients had been exposed to cardiothoracic surgical procedures, during which heater-cooler units (HCUs) were used.

Atypical mycobacteria can be detected in household water [8] and water-containing medical devices [9-11], and it had thus been suggested that the HCUs, which use water for thermoregulation during cardioplegia, might constitute the common source of the recent outbreak [12]. Indeed, an air-borne transmission of M. chimaera 


\section{FIGURE 1}

The functional set-up of an ECMO treatment unit, consisting of (A) an ECMO system; and (B) a thermoregulatory device at a medical intensive care unit, Homburg/Saar, Germany

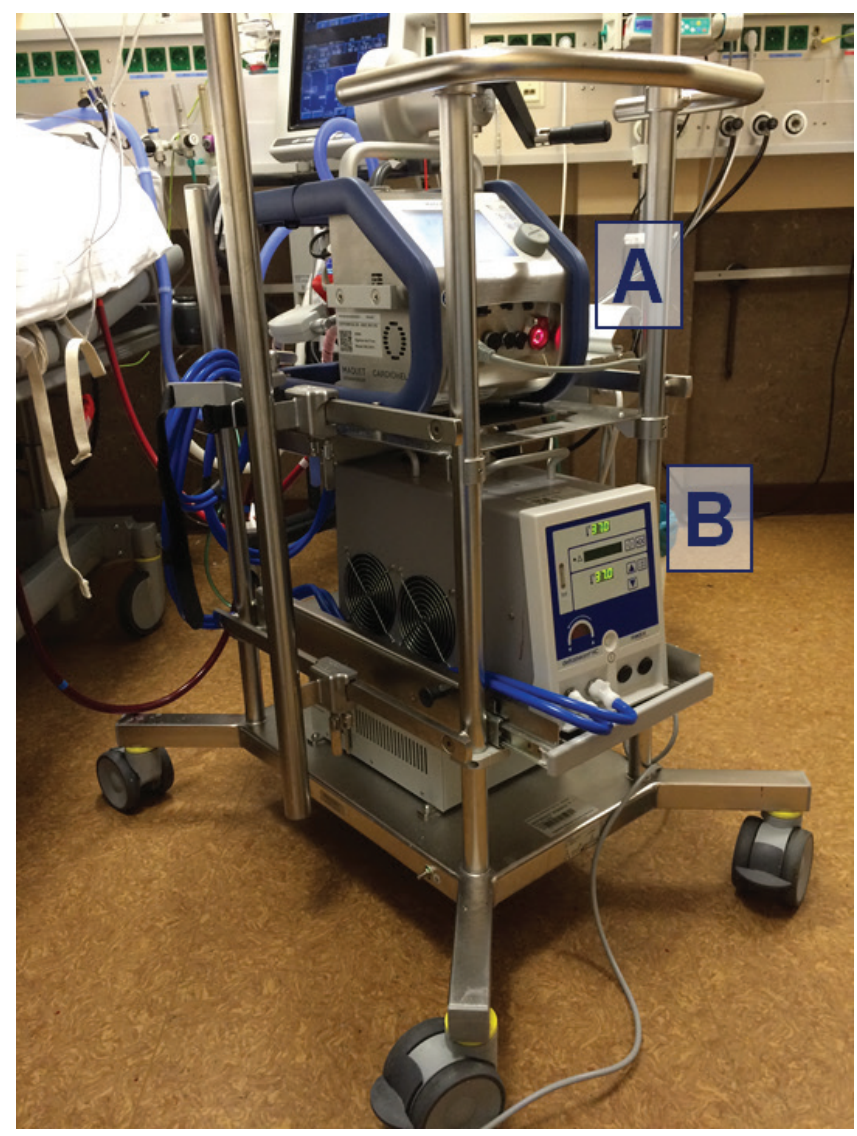

ECMO: extracorporeal membrane oxygenation.

in the operating room was confirmed [13] and a report by Haller et al. provided evidence that at least some of the HCUs might already have been contaminated at the manufacturing site [14]. Preventive measures to reduce the risk of transmission during cardiac surgery are now being implemented worldwide. Meanwhile, it remains to be elucidated whether other water-containing medical devices might also pose patients at risk of acquiring infections due to $M$. chimaera.

Veno-venous extracorporeal membrane oxygenation (ECMO) is an established treatment for patients with severe acute respiratory distress syndrome (ARDS) $[15,16]$. Additionally, veno-arterial circuit configurations offer a prolonged circulatory support, which is comparable to the short-term support provided by cardiopulmonary bypass (CPB) during cardiac surgery [17-20]. All extracorporeal circuits (ECC) consist of a tubing system, which is connected to a roller or centrifugal pump to maintain the active blood transport and guides the flow through the membrane oxygenator. Control units are used to adjust the blood flow between 2 and $7 \mathrm{~L}$ per minute. Thermoregulatory devices, heater units (HUs) or HCUs are engaged to adjust the blood temperature within the ECC [21]. All commercially available thermoregulatory systems run in analogy to those used in the operating room with circulating water. Hence, it may be hypothesised that ECMO treatment might also constitute a risk for transmission of water-borne pathogens. While patients treated with ECMO for respiratory failure have smaller potential entry sites for pathogens than those undergoing open-chest heart surgery, they are nevertheless critically ill and highly immunocompromised, and are thus susceptible to opportunistic infections. Additionally, as patients may be subjected to ECMO treatment for a prolonged duration of up to several months [22,23], there is a need to assess their potential exposure to water-borne pathogens such as $M$. chimaera in thermoregulatory devices used for ECMO.

Here, we present an in-depth assessment on the occurrence of $M$. chimaera in an ECMO centre in Germany, with a particular focus on potential mycobacterial transmission pathways and the clinical significance arising from our findings. Our investigation comprises two specific parts, i.e. (i) a prospective microbiological sampling of water from ECMO devices and from the environment for $M$. chimaera (August 2015-August 2016); and (ii) a retrospective patient chart analysis to identify potentially exposed individuals with positive M. chimaera culture results and previous ECMO treatment (April 2010-June 2016).

\section{Methods}

\section{Study site and study procedures}

The current study was carried out at the pneumological intensive care unit (ICU) at Saarland University Medical Center in Homburg, southwest Germany. This medical centre is a supra-regional ECMO centre and provides approximately 20 lung transplantations per year. Microbiological investigations pertaining to the presence of atypical mycobacteria in HCUs used during cardiac surgery were initiated in March 2015, and the prospective sampling of water from devices used for ECMO treatment was subsequently started in August 2015. Prompted by these microbiological investigations, a retrospective patient chart review of individuals treated with ECMO at our centre in the preceding 6 years was initiated in mid-2016 to further assess the significance of $M$. chimaera in this specific setting.

\section{Characteristics of extracorporeal circuits and thermoregulatory devices}

During the study period, veno-venous ECMO cannulation was performed using the femoral (draining) and jugular (return) veins as main cannula entry sites. As a standard, we used $23 \mathrm{~F}$ draining cannulae at a length of 38 or $55 \mathrm{~cm}$, as appropriate, and $19 \mathrm{~F}$ returning cannulae (Maquet Holding B.V. and Co. KG, Rastatt, Germany) with a heparin coating. Some patients underwent single stage cannulation using a bicaval double-lumen cannula (27 F or $31 \mathrm{~F}$ Avalon Elite, Avalon Laboratories; Rancho Dominguez, United States of 

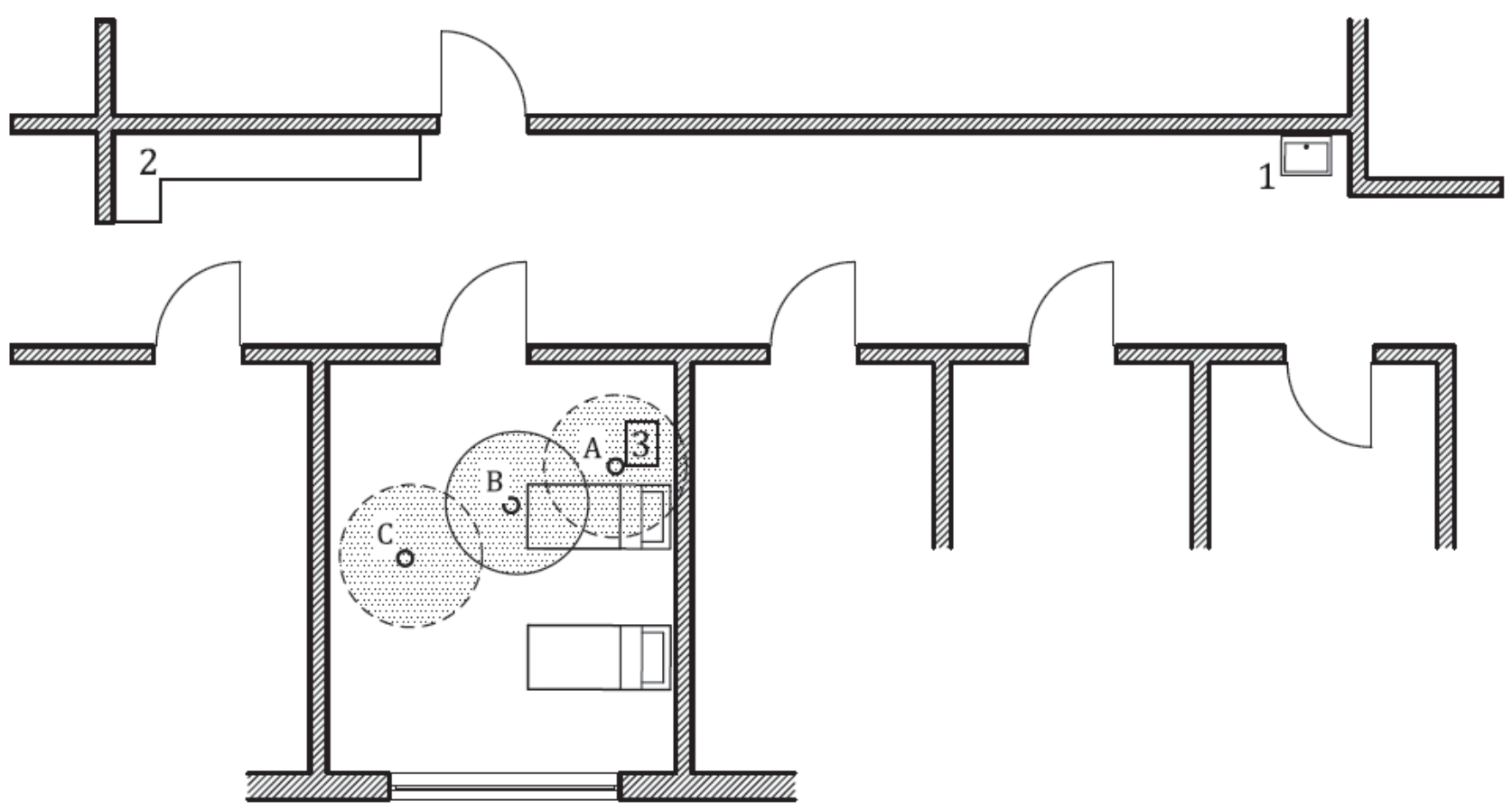

ECMO: extracorporeal membrane oxygenation.

The figure schematically shows the floor with the individual patient rooms where sampling took place. Water for thermoregulatory devices was taken from a sink (1) on the ward. The sink is equipped with a Pall Aquasafe filter to avoid microbial contamination. The filter is changed every 30 days, as recommended by the manufacturer. ECMO units are primed with sterile saline solution in the priming area (2). The ECMO machine and the thermoregulatory device are installed at the bedside (3) of the patient. Water samples were taken from the tap (1) and from the thermoregulatory devices. Air sampling took place (A) next to the ECMO device; (B) next to the patient; and (C) at 2-3 m distance from the patient and the ECMO device, but in the same room.

America). Standard oxygenators were $7.0 \mathrm{~L}-\mathrm{HLS}$ or Quadrox-I with a ROTAFLOW Centrifugal Pump RF 32 primed with physiological saline solution used on the Maquet CardioHelp or ROTAFLOW platform. Different thermoregulatory devices were used according to individual requirements; heater units such as 'Heater Unit HU 35' (Maquet) or HCUs such as 'Deltastream HC' (Medos Medizintechnik AG; Stolberg, Germany) and 'NovaTherm' (Novalung GmbH; Heilbronn, Germany). The functional set-up of an ECMO system with a heater unit is shown in Figure 1.

All thermoregulatory devices were temporarily leased from the manufacturers and filled with filtered tap

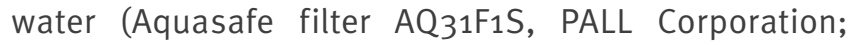
Dreieich, Germany; filter width: $0.2 \mu \mathrm{m})$. The priming of all circuits and corresponding thermoregulatory devices was performed within a central priming area and the devices were then placed bedside while being used. A schematic diagram of these operational areas is shown in Figure 2.
Processing and microbiological analysis of water from ECMO devices, tap water and environmental samples

For investigation of atypical mycobacteria, $100-250 \mathrm{~mL}$ of water were collected from the water tanks of thermoregulatory devices used for ECMO treatment, and were processed according to a protocol issued by the European Centre for Disease Prevention and Control (ECDC) [24]. In brief, water samples were concentrated by centrifugation and subsequently decontaminated using $\mathrm{N}$-acetyl-L-cysteine sodium hydroxide (NALC$\mathrm{NaOH}$ ). Additional examinations were carried out on filtered tap water (filter width $0.2 \mu \mathrm{m}$ ) that was used to fill the tanks of the ECMO devices. Of note, the tap water used is also regularly checked for compliance with the German drinking water directive [25]. Microscopy using auramine staining was carried out on all samples, and water samples were plated on two different media, i.e. (i) $7 \mathrm{H}_{11}$ Middlebrook agar; and (ii) Löwenstein-Jensen agar. Additionally, cultures in liquid media were also performed and water samples were inoculated into the MGIT 960 system. All culture media were obtained 
TABLE 1

Mycobacterial testing characteristics of water samples from thermoregulatory devices used for ECMO treatment at a pneumological intensive care unit, Germany, 2015-2016

\begin{tabular}{|c|c|c|c|c|c|c|}
\hline $\begin{array}{l}\text { Patient } \\
(\mathrm{n}=18)\end{array}$ & $\begin{array}{c}\text { Thermoregulatory } \\
\text { device }(n=10)\end{array}$ & $\begin{array}{l}\text { Device model } \\
\text { (Manufacturer) }\end{array}$ & Sampling date & Microscopy & Culture & $\begin{array}{c}\text { Species } \\
\text { identification }\end{array}$ \\
\hline 1 & 1 & Deltastream HC (Medos) & August 2015 & Positive $(+++)$ & Positive & M. chimaera \\
\hline 2 & 2 & Deltastream HC (Medos) & August 2015 & Negative & Positive & M. chimaera \\
\hline 3 & 3 & HU35 (Maquet) & December 2015 & Negative & Negative & - \\
\hline 4 & 4 & Deltastream HC (Medos) & January 2016 & Negative & Negative & - \\
\hline 5 & 5 & Deltastream HC (Medos) & January 2016 & Positive (+) & Positive & M. chimaera \\
\hline 6 & 4 & Deltastream HC (Medos) & January 2016 & Negative & Positive & M. chimaera \\
\hline 7 & 6 & Deltastream HC (Medos) & January 2016 & Negative & Negative & - \\
\hline 8 & 7 & HU35 (Maquet) & January 2016 & Negative & Negative & - \\
\hline 9 & 4 & Deltastream HC (Medos) & March 2016 & Positive (+) & Positive & $\begin{array}{l}\text { M. chimaera and } \\
\text { M. gordonae }\end{array}$ \\
\hline 10 & 8 & NovaTherm (NovaLung) & March 2016 & Negative & Negative & - \\
\hline 11 & 9 & Deltastream HC (Medos) & March 2016 & Negative & Negative & - \\
\hline 12 & 4 & Deltastream HC (Medos) & March 2016 & Positive $(+++)$ & Positive & M. chimaera \\
\hline 13 & 6 & Deltastream HC (Medos) & March 2016 & Negative & Negative & - \\
\hline 14 & 9 & Deltastream HC (Medos) & April 2016 & Negative & Negative & - \\
\hline 15 & 6 & Deltastream HC (Medos) & April 2016 & Negative & Positive & M. chimaera \\
\hline 16 & 8 & NovaTherm (Novalung) & April 2016 & Negative & Positive & M. chimaera \\
\hline 17 & 9 & Deltastream HC (Medos) & August 2016 & Positive (+ +) & Positive & M. chimaera \\
\hline 18 & 10 & Deltastream HC (Medos) & August 2016 & Negative & Negative & - \\
\hline
\end{tabular}

ECMO: extracorporeal membrane oxygenation; M: Mycobacterium.

For microscopy of auramine-stained slides, the following semi-quantitative grading scheme was adopted: (i) negative (no mycobacteria seen on the microscope slide); (ii) + (up to 50 mycobacteria seen per 100 observation fields); (iii) ++ (5-50 mycobacteria seen per 10 observation fields); and (iv) +++ ( $\geq 5$ mycobacteria seen per observation field).

from Becton Dickinson (Heidelberg, Germany) and were incubated for up to 8 weeks.

Environmental room air sampling was carried out in patient rooms during ECMO treatment while thermoregulatory devices, which had previously tested positive for M. chimaera, were running. For each sampling, 100$200 \mathrm{~L}$ of room air was collected using the MBASS 30 microbiological air sampling system (Umweltanalytik Holbach GmbH; Holbach, Germany) and conducted over selective $7 \mathrm{H}_{10}$ Middlebrook agar plates during one minute. During each sampling, air specimens were taken at three different locations, i.e. (i) next to the ECMO device; (ii) next to the patient; and (iii) in 2-3 $\mathrm{m}$ distance from the patient and the ECMO device, but in the same room. Additionally, swabs (eSwab, Copan Diagnostics; Brescia, Italy) were taken once from the surface and connecting tubes of selected ECMO thermoregulatory devices, and were subsequently analysed for the presence of mycobacteria. All agar plates were examined twice weekly during eight weeks for signs of mycobacterial growth. Suspicious colonies were identified to the species level using a commercially available molecular typing system (GenoType NTM-DR, Hain Lifescience; Nehren, Germany). Additionally, a subsample of positive specimens was sent to a reference centre for molecular diagnostics at the University Hospital Regensburg, Germany, where the species identification of $M$. chimaera was confirmed by partial sequencing of the $16 \mathrm{~S}$, ITS and $r p o B$ gene sequences.

Retrospective patient analysis and microbiological work-up of patient samples Using an electronic database, we retrospectively identified all patients undergoing ECMO treatment (excluding extracorporeal $\mathrm{CO}_{2}$ removal; $\mathrm{ECCO}_{2} \mathrm{R}$ ) at the pneumological ICU at Saarland University Medical Center between April 2010 and June 2016.

Respiratory samples were taken if clinical signs and symptoms of respiratory infection were present and/ or whenever a potential infection was clinically suspected. Bronchial specimens obtained during or after ECMO treatment were reviewed both clinically and microbiologically for findings suggestive of mycobacterial infection.

Due to the retrospective nature of the analysis, no specific protocol was implemented before the start of the study for the microbiological work-up of patient samples. Standard diagnostic procedures were followed and bronchial aspirates and bronchoalveolar lavage specimens of patients undergoing ECMO treatment were immediately sent to the microbiology laboratory using a pneumatic transport system. Upon receipt at the laboratory, samples were decontaminated using 
Characteristics and clinical course of patients diagnosed with Mycobacterium chimaera in respiratory specimens while treated with ECMO at a pneumological intensive care unit, Germany, 2010-2016 (n=3)

\begin{tabular}{|c|c|c|c|c|c|c|c|c|}
\hline $\begin{array}{l}\text { Patient } \\
\text { number }\end{array}$ & Sex & $\begin{array}{c}\text { Age } \\
\text { (years) }\end{array}$ & $\begin{array}{l}\text { Underlying disease and } \\
\text { operative intervention }\end{array}$ & $\begin{array}{l}\text { Indication for } \\
\text { ECMO }\end{array}$ & $\begin{array}{l}\text { Time of ECMO } \\
\text { treatment } \\
\text { (days) }\end{array}$ & $\begin{array}{l}\text { Risk factor } \\
\text { for } M \text {. } \\
\text { chimaera }\end{array}$ & $\begin{array}{c}\text { Days from ECMO } \\
\text { treatment onset } \\
\text { to sampling for } \\
\text { M. chimaera }\end{array}$ & Clinical course \\
\hline 1 & Male & Mid $70 \mathrm{~s}$ & CTEPH, PEA and CABG & ARDS & 48 & $\begin{array}{l}\text { Previous } \\
\text { open-chest } \\
\text { cardiac } \\
\text { surgery } \\
\end{array}$ & 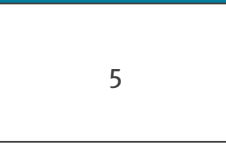 & $\begin{array}{c}\text { Died on ECMO } \\
\text { (cardiogenic } \\
\text { shock) }\end{array}$ \\
\hline 2 & Male & End $20 \mathrm{~s}$ & $\begin{array}{c}\text { AML, } \\
\text { allogenic SCT }\end{array}$ & GVHD & 113 & None & 6 & $\begin{array}{l}\text { Died on ECMO } \\
\text { (septic shock) }\end{array}$ \\
\hline 3 & Female & $\begin{array}{l}\text { Early } \\
305\end{array}$ & CF, LTx, CLAD & ARDS & 40 & $\begin{array}{l}\text { Previous } \\
\text { open-chest } \\
\text { cardiac } \\
\text { surgery }\end{array}$ & 205 & $\begin{array}{l}\text { Survived } \\
\text { (re-LTx) }\end{array}$ \\
\hline
\end{tabular}

AML: acute myeloid leukaemia; ARDS: acute respiratory distress syndrome; CABG: coronary artery bypass grafting; CF: cystic fibrosis; CLAD: chronic lung allograft dysfunction; CTEPH: chronic thromboembolic pulmonary hypertension; ECMO: extracorporeal membrane oxygenation; GVHD: graft vs. host disease; LTx: lung transplantation; PEA: pulmonary endarterectomy; re-LTX: lung retransplantation; SCT: stem cell transplantation.

NALC-NaOH and mycobacterial examinations were carried out as follows: (i) microscopy using Kinyoun or auramine staining; (ii) MGIT 960 liquid media culture; and (iii) culture on solid agar media (Löwenstein-Jensen agar; Stonebrink agar). All patient samples were incubated for up to 8 weeks and mycobacteria were identified as described above.

Species identification of $M$. chimaera had not uniformly been performed between 2010 and 2015, thus all isolates that had previously been identified as either $M$. intracellulare or $M$. avium were re-cultured from a biobank and subjected to molecular genetic testing for unambiguous species identification.

\section{Results}

\section{Detection of Mycobacterium chimaera in water and air samples}

Between August 2015 and August 2016, a total of 18 water samples originating from 10 different thermoregulatory devices used for ECMO treatment were subjected to microbiological analyses. M. chimaera was detected in nine specimens i.e. half of all examined water samples. Of the ten analysed thermoregulatory devices, water obtained from seven tested positive in at least one sample. In five water samples, mycobacteria were visible by microscopy, which suggests presence of a relatively high number of mycobacteria. The liquid medium culture (MGIT) was earliest to give positive results in all cases, after approximately 2 weeks of incubation (10-16 days). In one water sample which tested positive for $M$. chimaera, a co-colonisation with M. gordonae was observed. Details on the microbiological test results are given in Table 1.

Filtered tap water, which was commonly used to fill the thermoregulatory devices for ECMO treatment, was subjected to microbiological examinations at three different time points (several weeks apart), but neither bacterial nor mycobacterial pathogens were detected.

When analysing nine room air samples from the pneumological ICU, no atypical mycobacteria and no non-fermentative Gram-negative rods were detected during 8 weeks of incubation. Of note, some specimens contained low quantities of environmental Gram-positive (e.g. Arthrobacter spp., Bacillus subtilis, Corynebacterium amycolatum and Micrococcus luteus) and Gram-negative bacteria (e.g. Moraxella osloensis). Environmental moulds (e.g. Penicillium citrinum) were also found.

A series of 12 swabs taken from different surfaces and connecting tubes of two running ECMO thermoregulatory devices remained uniformly negative for $M$. chimaera.

\section{Occurrence of Mycobacterium chimaera in patients treated with ECMO}

We reviewed the electronic charts of all 118 patients who had received ECMO support between April 2010 and June 2016. Bronchial specimens (bronchial aspirates and/or bronchoalveolar lavage samples) from 79 patients $(67.0 \%)$ were analysed for the presence of mycobacteria during or after ECMO therapy. All patients received respiratory ECMO support either due to severe ARDS or as a temporary 'bridging procedure' to planned lung transplantation. A total of 32 of 79 (40.5\%) patients were male and the mean age was 46.8 years (standard deviation (SD): 16.7 years). The mean duration of ECMO treatment was 20.2 days (SD: 46.6 days), and $58.3 \%$ of the analysed patients survived to discharge. 
Mycobacteria were observed upon microscopy (auramine staining) in bronchial specimens from one individual among the 79 patients. In three cases, mycobacterial cultures were bacterially contaminated and could not be analysed, thus leading to a final cohort of 76 patients with mycobacterial culture results after the onset of ECMO treatment. Cultures for mycobacteria were positive in four patients and the mycobacterial species were identified as $M$. chimaera in three and $M$. malmoense in one of them. The three cases of $M$. chimaera were critically reviewed to investigate the possible clinical significance of this finding. Brief descriptions on the patient characteristics are given below and in Table 2.

\section{Patient 1}

In 2010, a man in his mid-70s developed severe acute respiratory distress syndrome (ARDS) and received ECMO therapy after pulmonary endarterectomy and coronary artery bypass grafting had been performed as treatment for chronic thromboembolic pulmonary hypertension and coronary heart disease. Eight days before ECMO initiation, the patient was screened for mycobacteria and was negative. On day 5 with ECMO support, a bronchial specimen was obtained that yielded $M$. chimaera. The patient died in cardiogenic shock after 48 days of ECMO treatment.

\section{Patient 2}

In 2013, a man in his late 20 s underwent allogenic stem cell transplantation for acute myeloid leukaemia, which he developed after treatment of Hodgkin's lymphoma with thymic infiltration. The patient developed graft vs. host disease with pulmonary involvement. Due to progressive respiratory failure, he was treated with ECMO with the intention to bridge the time to lung transplantation. After 6 days with ECMO, a bronchial aspirate was sent to the laboratory and $M$. chimaera was found once, but not in follow-up examinations 2 and 4 weeks later. Some weeks later, the patient was temporarily treated with clarithromycin, rifampicin, ethambutol and moxifloxacin for a clinically suspected mycobacterial infection. The patient died 113 days after initiation of ECMO therapy in septic shock with bacteraemia due to Enterococcus faecium.

\section{Patient 3}

A woman in her 30 s with cystic fibrosis developed a restrictive chronic allograft dysfunction with consecutive lung failure after previous lung transplantation. Hence, she was treated with ECMO in October 2014 and re-transplanted after 40 days with extracorporeal support. She had multiple bronchial aspirates being sampled for the presence of mycobacteria before ECMO (last sampling 11 days earlier) that were always negative. Two hundred five days after ECMO initiation, $M$. chimaera was detected in a bronchial aspirate. The patient is still alive (>650 days) and her clinical condition is good.

\section{Discussion}

In the present single-centre study, M. chimaera was detected in a considerable amount of water samples taken from different thermoregulatory devices of two different providers during ECMO treatment. Indeed, half of all analysed specimens grew $M$. chimaera, whereas no mycobacteria were found in room air samples and swabs from ECMO system surfaces. M. chimaera was also detected in three ECMO patients in a retrospective analysis over 6 years, but the transmission pathways as well as the clinical relevance of the findings remain uncertain.

M. chimaera was recently described as the causative agent in a multi-country outbreak of severe invasive infections, and pathogen transmission likely occurred through contaminated HCUs used during cardiac surgery $[4,5,7,14]$. By acknowledging the aetiological role of $M$. chimaera in this outbreak, its clinical relevance had to be reconsidered because previous studies had described $M$. chimaera to be of rather low pathogenicity [26]. Indeed, an analysis of 97 culture isolates from German patients detected a clinical relevance in merely $3.3 \%$ of all samples [2], and there is only a limited number of case reports providing evidence of infections due to $M$. chimaera in immunocompromised patients, such as those with severe anorexia nervosa [27], chronic obstructive pulmonary disease [28] and cystic fibrosis [29]. The patients on ECMO treatment described in our report were also immunocompromised and might thus have been at risk of clinical $M$. chimaera infection.

M. chimaera is able to form biofilms and may persist in water samples [8], which may partially explain its longlasting occurrence in water-containing HCUs used for open-chest cardiac surgery $[4,6]$. While device contamination during the production process [14] and a subsequent air-borne transmission [13,30,31] have been proposed as transmission pathways for this recent outbreak, the clinical significance of our findings in ECMO devices and the potential risks for patients remain to be elucidated. However, several characteristics seen in our study differ from those observed in connection with cardiac surgery. First, $M$. chimaera was detected in water from two different providers of thermoregulatory devices, thus rendering contamination during the production process of a single, specific device relatively unlikely. Second, an air-borne transmission of $M$. chimaera from the ECMO device to the patient could not be demonstrated. The ECMO-related thermoregulatory devices are, in contrast to HCUs used during cardiac surgery, air-tight and closed systems. In line with this, we did not find any evidence of detectable mycobacteria upon air sampling in patient rooms during ECMO treatment.

It is important to note that the mere diagnosis of an atypical mycobacterium in a bronchial specimen is not necessarily linked to an ongoing infection [32]. Indeed, following careful retrospective patient chart assessment in our study, we consider the detection of 
M. chimaera in bronchial aspirates from three patients during or after ECMO treatment not to be evidently associated with the $M$. chimaera contamination of the thermoregulatory devices. Patient 2 of the aforementioned patients, who was highly immunocompromised, suffered from pulmonary graft vs. host disease after allogenic SCT and had not been tested for atypical mycobacteria before ECMO therapy. Thus, it cannot be excluded that he might have already been colonised with atypical mycobacteria before ECMO treatment. In contrast, Patients 1 and 3 had been negative in mycobacterial sputum analyses before ECMO initiation. However, both patients had also been exposed to HCUs during open-chest surgery. Further molecular diagnostics could have helped to further characterise the origin of the $M$. chimaera strains found in these patients, e.g. through molecular analyses comparing their genetic characteristics to those of $M$. chimaera strains detected in water from ECMO devices and HCUs used in cardiac surgery.

ECMO is a life-saving technology, in particular for patients with severe respiratory failure despite maximal medical treatment $[16,33,34]$. Such patients suffer from comorbidities, are frequently immunocompromised and thus a highly vulnerable population. We therefore recommend that specific investigations for $M$. chimaera should be carried out in more ECMO centres to identify whether this pathogen constitutes a potentially relevant infectious agent in ECMO treatment settings. In our study, we were unable to identify a distinct source of the M. chimaera contamination. No mycobacteria were found in the tap water used to fill the thermoregulatory devices, thus rendering a contamination with environmental mycobacteria unlikely. A contamination during the manufacturing process of the thermoregulatory devices cannot be excluded, but seems rather unlikely because devices of different manufacturers were affected. Additionally, cross-contamination from cardiac HCUs used in the operating theatre might also have occurred, e.g. when surgery was performed on ECMO patients and the same oxygenator was used on different thermoregulatory devices.

Our study has several limitations. First, it is a singlecentre study with a limited sample size. Yet, our report is the first systematic assessment of $M$. chimaera beyond the setting of cardiac surgery, and therefore provides important additional evidence. Second, our clinical patient analysis is retrospective, mainly due to the fact that we initiated the current study only after the publication of the first outbreak reports related to $\mathrm{HCU}$ devices used during cardiac surgery. The retrospective design of our patient chart review might have biased some of our results, specifically pertaining to repeated sampling procedures for $M$. chimaera. Future research on this topic should thus preferably employ a prospective study design. Third, repeated sampling of water from thermoregulatory devices might have further improved the detection rate and more sophisticated microbiological analyses e.g. whole-genome sequencing and comparison of $M$. chimaera isolates obtained from water and patient samples could have elucidated the genetic relatedness of the mycobacterial strains. Fourth, additional microbiological investigations of all water samples pertaining to e.g. Legionella spp. and Pseudomonas spp. might have helped to better assess the water quality and to better quantify the contamination of the thermoregulatory devices.

\section{Conclusions}

Patients receiving ECMO treatment are often highly immunocompromised and prone to opportunistic infections, including those caused by atypical mycobacteria. The detection of $M$. chimaera in a considerable amount of water samples from thermoregulatory ECMO devices in our centre should encourage further research in other hospital centres to elucidate the origin of such contamination. Additionally, the hitherto unclear clinical relevance of $M$. chimaera in the setting of ECMO treatment needs to be assessed. Strict adherence to disinfection protocols published by the manufacturers of thermoregulatory devices as well as continued microbiological surveillance for $M$. chimaera are recommended to minimise the risk of infection.

\section{Acknowledgements}

We thank Dr Alexander Halfmann and the laboratory technicians Diana Velten, Susanne Loibl and Richard Schaum for excellent support during microbiological examinations. We express our gratitude to Monika Flaig for her assistance with retrospective patient chart analysis and to Elisabeth Trudzinski for provision of Figure 2.

\section{Conflict of interest}

None declared.

Authors' contributions

Specimen sampling: FCT, US, AK, KH, PML, SLB. Microbiological diagnostics: US, UR, BG, MH, SLB. Clinical patient chart review: FCT, PML. Patient treatment: FCT, AK, HW, RB, PML. Wrote the manuscript: FCT, PML, SLB. All authors have read and approved the final version of the manuscript.

\section{References}

1. Tortoli E, Rindi L, Garcia MJ, Chiaradonna P, Dei R, Garzelli C, et al. Proposal to elevate the genetic variant MAC-A, included in the Mycobacterium avium complex, to species rank as Mycobacterium chimaera sp. nov. Int J Syst Evol Microbiol. 2004;54(Pt 4):1277-85. DOI: 10.1099/ijs.0.02777-o PMID: 15280303

2. Schweickert B, Goldenberg O, Richter E, Göbel UB, Petrich $A$, Buchholz $P$, et al. Occurrence and clinical relevance of Mycobacterium chimaera sp. nov., Germany. Emerg Infect Dis. 2008;14(9):1443-6. DOI: 10.3201/eid1409.071032 PMID: 18760016

3. Boyle DP, Zembower TR, Reddy S, Qi C. Comparison of clinical features, virulence, and relapse among Mycobacterium avium complex species.Am J Respir Crit Care Med. 2015;191(11):13107. DOI: 10.1164/rccm.201501-00670C PMID: 25835090

4. Sax H, Bloemberg G, Hasse B, Sommerstein R, Kohler P, Achermann Y, et al. Prolonged outbreak of Mycobacterium 
chimaera infection after open-chest heart surgery. Clin Infect Dis. 2015;61(1):67-75. DOI: 10.1093/cid/civ198 PMID: 25761866

5. Achermann Y, Rössle M, Hoffmann M, Deggim V, Kuster S, Zimmermann DR, et al. Prosthetic valve endocarditis and bloodstream infection due to Mycobacterium chimaera. J Clin Microbiol. 2013;51(6):1769-73. DOI: 10.1128/JCM.00435-13 PMID: 23536407

6. Kohler P, Küster SP, Bloemberg G, Schulthess B, Frank M, Tanner FC, et al. Healthcare-associated prosthetic heart valve, aortic vascular graft, and disseminated Mycobacterium chimaera infections subsequent to open heart surgery. Eur Heart J. 2015;36(40):2745-53. DOI: 10.1093/eurheartj/ehv342 PMID: 26188001

7. Tan N, Sampath R, Abu Saleh OM, Tweet MS, Jevremovic D, Alniemi S, et al. Disseminated Mycobacterium chimaera infection after cardiothoracic surgery. Open Forum Infect Dis. 2016;3(3):ofw131. DOI: 10.1093/ofid/ofw131 PMID: 27703994

8. Wallace RJ, lakhiaeva E, Williams MD, Brown-Elliott BA, Vasireddy S, Vasireddy R, et al. Absence of Mycobacterium intracellulare and presence of Mycobacterium chimaera in household water and biofilm samples of patients in the United States with Mycobacterium avium complex respiratory disease. J Clin Microbiol. 2013;51(6):1747-52. DOI: 10.1128/JCM.00186-13 PMID: 23536397

9. Makovcova J, Slany M, Babak V, Slana I, Kralik P. The water environment as a source of potentially pathogenic mycobacteria.J Water Health. 2014;12(2):254-63. DOI: $10.2166 /$ wh.2013.102 PMID: 24937219

10. Garvey MI, Ashford R, Bradley CW, Bradley CR, Martin TA, Walker J, et al. Decontamination of heater-cooler units associated with contamination by atypical mycobacteria. J Hosp Infect. 2016;93(3):229-34. DOI: 10.1016/j. jhin.2016.02.007 PMID: 27112044

11. Kanamori H, Weber DJ, Rutala WA. Healthcare outbreaks associated with a water reservoir and infection prevention strategies.Clin Infect Dis. 2016;62(11):1423-35. DOI: 10.1093/ cid/ciw122 PMID: 26936670

12. Götting T, Klassen S, Jonas D, Benk Ch, Serr A, Wagner D, et al. Heater-cooler units: contamination of crucial devices in cardiothoracic surgery. J Hosp Infect. 2016;93(3):223-8. DOI: 10.1016/j.jhin.2016.02.006 PMID: 27101883

13. Sommerstein R, Rüegg C, Kohler P, Bloemberg G, Kuster SP, $\mathrm{Sax} \mathrm{H}$. Transmission of Mycobacterium chimaera from heatercooler units during cardiac surgery despite an ultraclean air ventilation system.Emerg Infect Dis. 2016;22(6):1008-13. DOI: 10.3201/eid2206.160045 PMID: 27070958

14. Haller S, Holler C, Jacobshagen A, Hamouda O, Abu Sin M, Monnet DL, et al. Contamination during production of heatercooler units by Mycobacterium chimaera potential cause for invasive cardiovascular infections: results of an outbreak investigation in Germany, April 2015 to February 2016. Euro Surveill. 2016;21(17):30215.

15. Brodie D, Bacchetta M. Extracorporeal membrane oxygenation for ARDS in adults. N Engl J Med. 2011;365(20):1905-14. DOI: 10.1056/NEJMct1103720 PMID: 22087681

16. Aokage T, Palmér K, Ichiba S, Takeda S. Extracorporeal membrane oxygenation for acute respiratory distress syndrome.J Intensive Care. 2015;3(1):17. DOI: 10.1186/s40560015-0082-7 PMID: 27408728

17. Bakhtiary F, Keller H, Dogan S, Dzemali O, Oezaslan F, Meininger D, et al. Venoarterial extracorporeal membrane oxygenation for treatment of cardiogenic shock: clinical experiences in 45 adult patients. J Thorac Cardiovasc Surg. 2008;135(2):382-8. DOI: 10.1016/j.jtcvs.2007.08.007 PMID: 18242273

18. Rastan AJ, Dege A, Mohr M, Doll N, Falk V, Walther T, et al. Early and late outcomes of 517 consecutive adult patients treated with extracorporeal membrane oxygenation for refractory postcardiotomy cardiogenic shock. J Thorac Cardiovasc Surg. 2010;139(2):302-11, 311.e1. DOI: 10.1016/j. jtcvs.2009.10.043 PMID: 20106393

19. Mirabel M, Luyt CE, Leprince $P$, Trouillet JL, Léger $P$, Pavie A, et al. Outcomes, long-term quality of life, and psychologic assessment of fulminant myocarditis patients rescued by mechanical circulatory support. Crit Care Med. 2011;39(5):1029-35. DOI: 10.1097/CCM.obo13e31820ead 45 PMID: 21336134

20. Werdan K, Gielen S, Ebelt H, Hochman JS. Mechanical circulatory support in cardiogenic shock. Eur Heart J. 2014;35(3):156-67. DOI: 10.1093/eurheartj/eht248 PMID: 24014384

21. Squiers JJ, Lima B, DiMaio JM. Contemporary extracorporeal membrane oxygenation therapy in adults: Fundamental principles and systematic review of the evidence.J Thorac Cardiovasc Surg. 2016;152(1):20-32. DOI: 10.1016/j. jtcvs.2016.02.067 PMID: 27060027
22. Hoopes CW, Kukreja J, Golden J, Davenport DL, Diaz-Guzman $\mathrm{E}$, Zwischenberger JB. Extracorporeal membrane oxygenation as a bridge to pulmonary transplantation.J Thorac Cardiovasc Surg. 2013;145(3):862-7, discussion 867-8. DOI: 10.1016/j. jtcvs.2012.12.022 PMID: 23312979

23. Kon ZN, Wehman PB, Gibber M, Rabin J, Evans CF, Rajagopal K, et al. Venovenous extracorporeal membrane oxygenation as a bridge to lung transplantation: successful transplantation after 155 days of support. Ann Thorac Surg. 2015;99(2):704-7. DOI: 10.1016/j.athoracsur.2014.04.097 PMID: 25639416

24. European Centre for Disease Prevention and Control (ECDC). ECDC Technical Document. EU protocol for case detection, laboratory diagnosis and environmental testing of Mycobacterium chimaera infections potentially associated with heater-cooler units: case definition and environmental testing methodology. Stockholm: ECDC. 2015. [Accessed 17 Oct 2016]. Available from: http://ecdc.europa.eu/en/publications/ Publications/EU-protocol-for-M-chimaera.pdf

25. Verordnung über die Qualität von Wasser für den menschlichen Gebrauch (Trinkwasserverordnung - TrinkwV 2001), Neufassung vom 10. März 2016. 2016. [German ordinance on the quality of water intended for human consumption (Trinkwasserverordnung - TrinkwV 2001) as published on 10 March 2016]. [Accessed 1 Nov 2016]. German. Available from: http://www.bgbl.de/xaver/bgbl/start. xav?startbk=Bundesanzeiger_BGBl\&jumpTo=bgbl116so 459 . pdf\#_bgbl__\%2F\%2F*\%5B\%40attr_id\%3D\%27bgbl116s0 459. pdf\%27\%5D 1478020685560 [in German]

26. Moon SM, Kim SY, Jhun BW, Lee H, Park HY, Jeon K, et al. Clinical characteristics and treatment outcomes of pulmonary disease caused by Mycobacterium chimaera. Diagn Microbiol Infect Dis. 2016;S0732-8893(16)30315-7.PMID: 27720208

27. Alhanna J, Purucker M, Steppert C, Grigull-Daborn A, Schiffel G, Gruber H, et al. Mycobacterium chimaera causes tuberculosis-like infection in a male patient with anorexia nervosa. Int J Eat Disord. 2012;45(3):450-2. DOI: 10.1002/ eat.20942 PMID: 21656541

28. Bills ND, Hinrichs SH, Aden TA, Wickert RS, Iwen PC. Molecular identification of Mycobacterium chimaera as a cause of infection in a patient with chronic obstructive pulmonary disease.Diagn Microbiol Infect Dis. 2009;63(3):292-5. DOI: 10.1016/j.diagmicrobio.2008.12.002 PMID: 19216940

29. Cohen-Bacrie S, David M, Stremler N, Dubus JC, Rolain JM, Drancourt M. Mycobacterium chimaera pulmonary infection complicating cystic fibrosis: a case report.J Med Case Reports. 2011;5(1):473. DOI: 10.1186/1752-1947-5-473 PMID: 21939536

30. Perkins KM, Lawsin A, Hasan NA, Strong M, Halpin AL, Rodger $\mathrm{RR}$, et al. Notes from the field: Mycobacterium chimaera contamination of heater-cooler devices used in cardiac surgery - United States. MMWR Morb Mortal Wkly Rep. 2016;65(40):1117-8. DOI: 10.15585/mmwr.mm6540a6 PMID: 27740609

31. Schreiber PW, Küster SP, Hasse B, Bayard C, Rüegg C, Kohler $P$, et al. Reemergence of Mycobacterium chimaera in heatercooler units despite intensified cleaning and disinfection protocol. Emerg Infect Dis. 2016;22(10):1830-3. DOI: 10.3201/ eid2210.160925 PMID: 27649345

32. van Ingen J. Microbiological diagnosis of nontuberculous mycobacterial pulmonary disease.Clin Chest Med. 2015;36(1):43-54. DOI: 10.1016/j.ccm.2014.11.005 PMID: 25676518

33. Peek GJ, Mugford M, Tiruvoipati R, Wilson A, Allen E, Thalanany MM, et al. , CESAR trial collaboration. Efficacy and economic assessment of conventional ventilatory support versus extracorporeal membrane oxygenation for severe adult respiratory failure (CESAR): a multicentre randomised controlled trial.Lancet. 2009;374(9698):1351-63. DOI: 10.1016/ S0140-6736(09)61069-2 PMID: 19762075

34. Noah MA, Peek GJ, Finney SJ, Griffiths MJ, Harrison DA, Grieve $\mathrm{R}$, et al. Referral to an extracorporeal membrane oxygenation center and mortality among patients with severe 2009 influenza $A\left(\mathrm{H}_{1} \mathrm{~N}_{1}\right)$. JAMA. 2011;306(15):1659-68. DOI: 10.1001/ jama.2011.1471 PMID: 21976615

\section{License and copyright}

This is an open-access article distributed under the terms of the Creative Commons Attribution (CC BY 4.0) Licence. You may share and adapt the material, but must give appropriate credit to the source, provide a link to the licence, and indicate if changes were made.

This article is copyright of the authors, 2016. 